\title{
Propertymanagement als Professional Service - Implikationen für die Outsourcing-Praxis
}

\author{
Sigrun Lüttringhaus
}

Eingegangen: 15. September 2015 / Angenommen: 5. Mai 2016 / Online publiziert: 6. Juni 2016 (C) The Author(s) 2016. This article is available at SpringerLink with Open Access

Zusammenfassung Assetmanager, die das Wertsteigerungspotenzial ihrer Immobilienportfolios während der Haltephase nutzen wollen, sind auf ein professionelles Propertymanagement ihrer Liegenschaften angewiesen. Letzteres wird häufig an einen Dienstleister outgesourct mit dem Ziel, Kosten einzusparen. Angestrebt werden insbesondere Skaleneffekte durch den Fremdbezug standardisierter Dienstleistungen, die anhand von zuvor festgelegten Service-Levels in Auftrag gegeben und gemessen werden. Dieser Ansatz greift in den meisten Fällen jedoch zu kurz, da das Propertymanagement eine doppelköpfige Dienstleistung ist, die nicht nur generic services umfasst, sondern aufgrund ihrer Management-Bestandteile vielmehr den Professional Services zuzurechnen ist. Die Propertymanagement-Dienstleistung ist wissenschaftlich bislang kaum erforscht. Diese Lücke sucht der vorliegende Beitrag zu schließen. Ziel ist eine theoretisch fundierte Konzeption des Propertymanagements als Professional Service. In einem ersten Schritt erfolgt eine kurze Erläuterung des Dienstleistungsbegriffes und speziell der Professional Services. Sodann wird das Propertymanagement als Teil des Immobilienmanagements verortet. Im Anschluss wird der Leistungskatalog des Propertymanagements dargestellt, und es erfolgt eine Abgrenzung zur Schwesterdisziplin Facilitymanagement. Schließlich wird die komplexe und personalintensive Propertymanagement-Dienstleistung als Professional Service identifiziert. Diese Klassifizierung hat weitreichende Konse-

Überarbeitete Fassung von Kapitel 2 „Propertymanagement als Professional Service“ aus:

Lüttringhaus, Sigrun: Outsourcing des Propertymanagements als Professional Service. Eine Analyse der Partnerwahlfaktoren. [Online-Edition: http://tuprints.ulb.tu-darmstadt.de/id/eprint/4420] TU

Darmstadt [Dissertation], (2015).

\footnotetext{
S. Lüttringhaus $(\bowtie)$

Forschungscenter Betriebliche Immobilienwirtschaft, TU Darmstadt, Hochschulstr. 1, Darmstadt, Deutschland

E-Mail: luettringhaus.sigrun@gmail.com

S. Lüttringhaus

Union Investment Real Estate GmbH, Valentinskamp 70 [EMPORIO], 20355 Hamburg, Deutschland
} 
quenzen für den gesamten Prozess des Fremdbezugs der Dienstleistung. Am Ende des Beitrags wird beispielhaft auf die Praxis-Implikationen für das Propertymanagement-Outsourcing und speziell die Partnerwahlphase eingegangen.

Schlüsselwörter Propertymanagement - Immobilienmanagement · Facilitymanagement $\cdot$ Professional Services $\cdot$ Dienstleistungen

Abstract In real estate investments such as open-ended real estate funds, value has traditionally been generated through transactions. This approach is now increasingly being complemented by employing value-added strategies during the holding period of a real estate investment. To implement those strategies, professional portfolio owners need professional managers.

While strategic value-added partnerships are well-established phenomenons in other industries, such as information technology, these partnerships remain scarce in real estate. Unlike asset management, property management is an operative task, and, depending on the client, it is often not even considered as a management activity. Therefore, property management has already been outsourced to external providers to a great extent applying the well-known criteria for "make-or-buy" decisions. The decision in favor of "buying" property management services therefore follows the same reasoning as for standardized commodities for which established markets exist.

Unfortunately, property management services often do not meet the aforementioned criteria: Rather than being mere commodities, these services have to be individually composed and executed given that context and goals of properties are specific and thus heterogenic. Against this backdrop, established outsourcing-procedures may not be translated one-to-one to outsourcing property management.

However, market participants do not seem to be fully aware of the particular challenge posed by outsourcing property management. Hence, standard procedures are still being used. Moreover, most purchasers look first and foremost at costs when outsourcing property management. In particular, they aim at generating economies of scale. Adopting a cost minimization strategy is based on the assumption that the quality of outsourced services can fully be defined by service levels. Whereas this may work out for most standard tasks, this assumption fails with regard to more important management tasks.

The core problem is that practitioners often fail to distinguish between two fundamentally different kinds of services that are the building blocks of "property management": Generic services, on the one hand, include e.g. bookkeeping, cleaning or repair works and are very different from management services, on the other hand, such as analyzing vacancies, budget planning, or awarding maintenance contracts. While the former services can be described, priced, outsourced and controlled with relative ease, the latter can only be partially tracked and controlled, especially when it comes to service quality. Moreover, quality assessment is rather individual and subjective, depending on the objectives and the context of the client. While the former services can be summarized as "commodity services", the latter, where individual knowledge, problem solving competency and ethics of the provider company are important, are called "professional services". 
The outsourcing of commodity services aims to increase efficiency and thus minimize costs. Subcontracting of professional services, in contrast, often targets to achieve a higher quality level. Higher quality usually goes along with higher cost, but the leverage, professional property managers can have on cost and return, is expected to make up many times for this higher cost. Put in business terms, higher competencies of employees, economies of scope and long-term relationships (i.e. knowing the specifics that are important) are crucial criteria for management services. Unlike commodity services these more sophisticated services may only partly be measured by service-level- agreements, as service level agreements do not guarantee effective communication and a successful relationship between the outsourcing partners or the service provider and the investor's tenants. Asset managers buying professional property management services usually expect management skills going above and beyond mastering routine administrative tasks. Yet, as these additional expectations cannot always be defined in advance, clients have to trust in the provider's abilities. However, there is a trade-off between superior management skills on the one and the cheapest price on the other hand.

It therefore does not come as a surprise that asset managers are not satisfied with their property managers: Several real estate management surveys have documented this phenomenon. In light of this, rather than solely focusing on cost, asset managers increasingly aim to find an outsourcing partner who may cater to their individual needs. Albeit this recent development, property management still remains uncharted territory in business studies literature.

This article aims to fill this void by developing a theory-based concept of property management as a professional service. In a first step, services and especially professional services are being defined. In a second step, property management is characterized as a real estate management service. In a third step, this paper illustrates the service-components of property management and the differences between property management and facility management. Finally, property management is identified as a professional service. Its characterization as a professional service has far-reaching consequences for the entire outsourcing process. An outlook on these consequences will be given at the end of the article.

Keywords Property management $\cdot$ Real estate management $\cdot$ Facility management $\cdot$ Professional Services $\cdot$ Generic services

\section{Einleitung}

Wertsteigerungspotenziale von Immobilienportfolios, zum Beispiel die von offenen Immobilienfonds, werden nicht mehr hauptsächlich durch schnelle Weiterveräußerung von Objekten realisiert, sondern vielmehr durch die Nutzung von Wertsteigerungsmechanismen während der Haltephase. Hierfür benötigen professionelle Bestandshalter ein professionelles Management ihrer Liegenschaften (Gibson, 1994, S. 9ff.). Gerade eine gute Zusammenarbeit zwischen Asset- und Propertymanagement ist wichtig, um eine gute Performance einer Immobilie zu erreichen (vgl. Salustri, 2016). Die verschiedenen Disziplinen des Immobilienmanagements und 
ihre Leistungsbestandteile, vor allem aber auch die Abgrenzung untereinander, sind Gegenstand wissenschaftlicher Diskussion (so Teichmann, 2007 und Kämpf-Dern, 2008 und Gondring u. Wagner, 2016), wobei zuweilen sogar konträre Konzeptionen des Propertymanagements vertreten werden. Hinzu kommt, dass - anders als in den Vereinigten Staaten mit Vereinigungen wie der Property Management Association oder der National Organization of Residential Property MANAGERS - bislang in Deutschland kein Verband für Propertymanagement existiert. Das Ringen um die Konkretisierung der Begrifflichkeiten ist dabei nicht auf Deutschland beschränkt, als Beispiel aus dem Ausland sind etwa die Richtlinien des Immobilienverbandes der ROYAL InSTITUTION OF CHARTERED SURVEYoRS (RICS) zu nennen (siehe RICS, 2011 und RICS, 2012 und RICS, 2016). In den sogenannten guidance notes geht es dabei immer wieder um die Definition und Inhalte der Managementdisziplinen in der Immobilienwirtschaft. Auch eine 2015 durchgeführte Studie des Institute of Real Estate Management (IREM) zeigt, dass die Grenzen zwischen den Aufgaben des Property- und Assetmanagements verschwimmen und in der Praxis unterschiedlich gezogen werden (Salustri, 2016, S.45).

Die Outsourcingquote des Propertymanagements lag 2009 bei 78,5\% (Wonneberger, 2009). Mit dem auf Basis einer Make-or-buy-Entscheidung getroffenen Fremdbezug des im Vergleich zum Assetmanagement weniger strategisch ausgerichteten Propertymanagements versprechen sich viele Auftraggeber in erster Linie Kosteneinsparungen. Angestrebt werden insbesondere Skaleneffekte durch den Fremdbezug standardisierter Dienstleistungen, die anhand von zuvor festgelegten ServiceLevels beauftragt und gemessen werden. Dieser Ansatz greift in den meisten Fällen jedoch zu kurz, da das Propertymanagement nur zum Teil aus einfachen Services und vor allem auch aus komplexen Management-Aufgaben besteht und daher der Dienstleistungskategorie der Professional Services zuzuordnen ist.

Immobilienwirtschaftliche Dienstleistungen sind bisher nur ansatzweise als Professional Service erfasst worden. Während KLOESS in seiner Untersuchung der Erfolgsfaktoren des Immobilienmanagements zwar schon einen „Professional approach" befürwortet, geht es ihm primär darum, dass Immobilien-ManagementDienstleister sich an den Werten und dem Selbstverständnis von Professional Service Firms, wie etwa Wirtschaftsprüfern, orientieren sollten (Kloess, 1999, S. 156). Kämpf-Dern hat demgegenüber nachgewiesen, dass das Real Estate Assetmanagement ein Professional Service ist (Kämpf-Dern, 2010). Das IREM untersucht in einem White-Paper das Zusammenspiel von Asset- und Propertymanagement und sieht mit Blick auf das Propertymanagement ,,an increased sophistication and more strategic alignment with asset management" (Salustri, 2016, S. 45).

Vor diesem Hintergrund leistet der vorliegende Beitrag Grundlagenarbeit und macht es sich zur Aufgabe, eine theoretisch fundierte Konzeption des Propertymanagements als Professional Service zu entwickeln. Dazu wird das Propertymanagement in den Kanon der immobilienwirtschaftlichen Dienstleistungen eingeordnet. Das Propertymanagement wird definiert, und es erfolgt eine Abgrenzung vom Facilitymanagement. Zweitens wird gezeigt, warum das Propertymanagement ein Professional Service ist. Schließlich werden Auswirkungen des Professional-ServiceCharakters für das Propertymanagement-Outsourcing aufgezeigt. 


\section{Definition und Leistungskatalog des Propertymanagements}

\subsection{Perspektiven und Ebenen des Immobilienmanagements}

In der Immobilienwirtschaft lassen sich die drei Blickrichtungen Nutzer-, Eigentümer- und Produzentenperspektive unterscheiden. (vgl. Pfnür, 2011, S.38). Diese Perspektiven sind mit unterschiedlichen Zielen verbunden: Während der Nutzer die Immobilie als Betriebsmittel betrachtet und das Verhältnis von Nutzen zu Kosten maximieren möchte, ist für den Eigentümer die Maximierung des in der Immobilie gebundenen Kapitals oberste Maxime. Ziel des Produzenten wiederum ist es, das Ergebnis aus der Bereitstellung von Sachgütern und Dienstleistungen zu optimieren (siehe Pfnür, 2011, S.24ff.). Das Propertymanagement ist eine Dienstleistung, welche die Eigentümerperspektive impliziert.

Aus diesen unterschiedlichen Zielsetzungen lassen sich die Aufgaben der verschiedenen Akteure rund um die Immobilie ableiten. Das Propertymanagement ist Teil des Immobilienmanagements, also der ,branchenspezifische(n) Führungslehre der Immobilienwirtschaft“ (Kämpf-Dern u. Pfnür, 2009, S.4). Die Aufgaben des Immobilienmanagements sind die Planung, Steuerung und Kontrolle ,der auf den Erfahrungsgegenstand Immobilie gerichteten ziel- und zweckabhängigen Transformationsprozesse“ (Kämpf-Dern u. Pfnür, 2009, S.4).

Das Immobilienmanagement untergliedert sich grundsätzlich in drei Ebenen, nämlich die Investment-, die Portfolio- und die Objektebene.

Auf jeder dieser Stufen lassen sich erneut die vorgenannten Perspektiven rund um die Immobilie einnehmen, das heißt, auch beim Immobilienmanagement existieren der nutzungsorientierte, der finanzwirtschaftliche und der immobilientechnologische Blickwinkel (Kämpf-Dern, 2009, S.3), so dass ein dreidimensionales Gefüge entsteht (siehe Abb. 1). Der Würfel zeigt die unterschiedlichen Dimensionen der verschiedenen Ebenen des Immobilienmanagements. So bilden die verschiedenen Managementebenen die verschiedenen Phasen des Immobilien-Lebenszyklus ab und können aus den drei genannten Perspektiven des Nutzers, des Anlegers und des Immobilienentwicklers durchgeführt oder in Auftrag gegeben werden. Der Würfel veranschaulicht das in der Einleitung angesprochene Aufbrechen der Wertschöp-

Abb. 1 Kern-Dimensionen des Immobilienmanagements (Kämpf-Dern u. Pfnür, 2009, S. 14.)

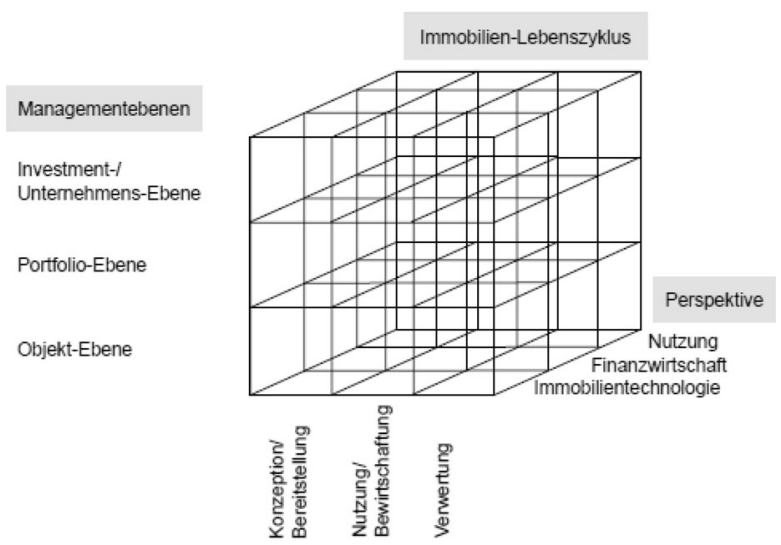


fungskette im Immobilienmanagement. Die Immobilienmanagement-Aufgaben werden nicht mehr strikt parallel zu den verschiedenen Phasen des Lebenszyklus einer Immobilie definiert und gestaltet, sondern vielmehr modular verstanden.

Während auf der höchsten Ebene, der Investmentebene, die Unternehmenspolitik in Bezug auf die immobilienwirtschaftlichen Aktivitäten festgelegt wird, werden auf der mittleren Ebene, der Portfolioebene, die strategischen Fragestellungen rund um das Portfolio verantwortet. Die unterste Ebene teilt sich in die strategische Objektebene einerseits und in die operative Objektebene andererseits. Das strategische Objektmanagement ist für die Umsetzung der Portfoliostrategie verantwortlich, indem es objektbezogene Strategien erstellt. Das operative Objektmanagement nimmt die operativen Managementaufgaben am Objekt wahr und steuert neben den eigenen Geschäftsprozessen auch die internen und externen Dienstleister (Kämpf-Dern u. Pfnür, 2009, S. 23f.).

\subsection{Die Dienstleistung Propertymanagement}

Abb. 2 zeigt zuoberst die verschiedenen Ebenen des Immobilienmanagements. In der Mitte sind die Lebenszyklusphasen einer Immobilie abgebildet und zuunterst die unterstützenden Real Estate Services. Das Propertymanagement ist unter dem Investment- sowie dem Portfolio- und dem Assetmanagement angesiedelt. Die Arbeit des Propertymanagers beginnt nach Abschluss des Projektmanagements beziehungsweise nach Bau der Immobilie und endet, wenn die Immobilie veräußert wird und das Transaktionsmanagement greift. Der Propertymanager arbeitet in der Bewirtschaftungsphase der Immobilie, welche Bestandsentwicklung, Instandhaltung und Vermietung einschließt, in enger Absprache mit dem Assetmanager zusammen. Zum Management der Immobilie arbeiten der Asset- und der Propertymanager mit Real Estate Services, wie technischen Dienstleistern, Maklern oder Marketingagenturen, zusammen.
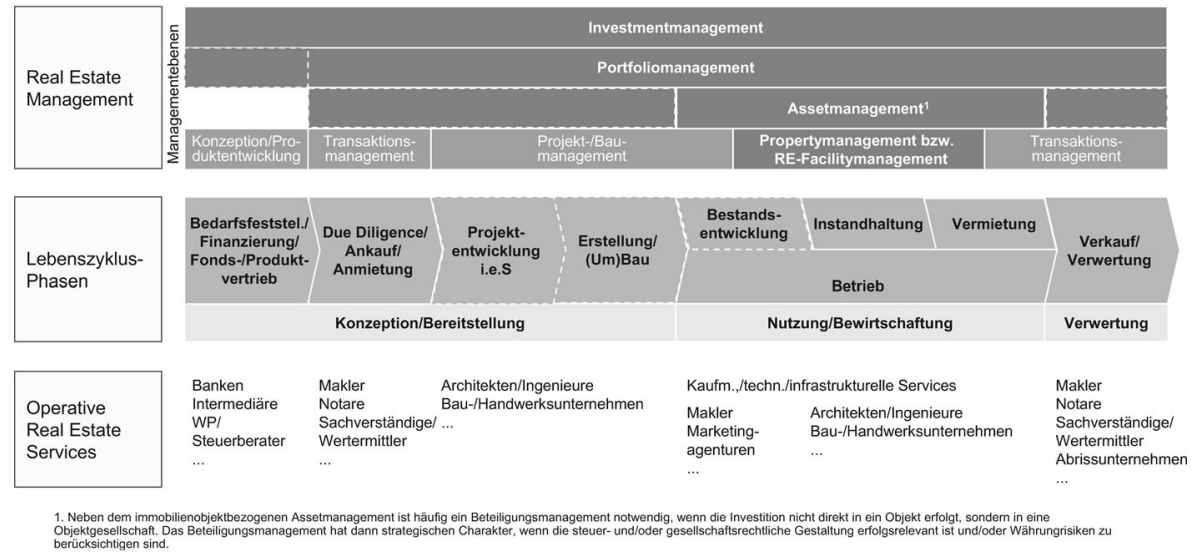

Abb. 2 Managementebenen, Lebenszyklusphasen und Geschäftsprozess-(Dienst-)Leistungen des Immobilienmanagements. (Kämpf-Dern u. Pfnür, 2009, S. 26.) 
Zwischen den Vertretern der drei beschriebenen Blickrichtungen - Nutzer, Eigentümer und Betreiber - bestehen Spannungen. So hat etwa der Investor einer Büroimmobilie ein vorgegebenes Budget einzuhalten, aus dem Zahlungen an den Dienstleister geleistet werden können. Der Mieter wiederum möchte beispielsweise seinen eigenen Nutzen in der Immobilie maximieren und fordert etwa Umbauten der Flächen durch den Investor/Eigentümer. Für den Dienstleister steht die Dienstleistung, hier das Propertymanagement, im Vordergrund. Für seine Leistung möchte er vom Eigentümer möglichst reich entlohnt werden. Gleichzeitig muss er aber auch den Mieter zufriedenstellen, um die Zufriedenheit des Eigentümers zu gewährleisten. „Zielsetzung des Property Managements ist die treuhänderische Eigentümervertretung sowie die Erreichung eines optimalen Preis-Leistungs-Verhältnisses der immobilienbezogenen Dienstleistungen [...] (Gondring u. Wagner, 2016, S. 13). Aufgrund der unterschiedlichen Interessenlage besteht zwischen den Parteien Konfliktpotenzial. Die Bedürfnisse des Mieters, seien es etwa renovierte, gut ausgestattete Büroräume, vertragen sich zum Beispiel nur bedingt mit dem Ziel des Eigentümers, die Renovierungskosten des Gebäudes möglichst gering zu halten. Ein möglicher Ausweg wäre eine Mieterhöhung. Darüber hinaus existieren in der Praxis Überschneidungen: So mögen etwa Eigentümer und Nutzer identisch sein.

Oeljeschlager führt an, dass zwar Eigentümer gleichzeitig Nutzer und Betreiber der Immobilie sein können, in der Regel dann aber innerhalb der Unternehmung entsprechende organisatorische Einteilungen vorhanden seien (Oeljeschlager, 2001, S. 255). Eine solche organisatorische Einteilung muss allerdings nicht zwingend verhindern, dass Zielkonflikte zwischen den Parteien beziehungsweise den Zielen existieren (zu den Koordinationsbeziehungen im betrieblichen Immobilienmanagement oder Corporate Real Estate Management siehe Pfnür, 2011, S. 273ff.). Darüber hinaus können mehrere Dienstleister beteiligt sein, woraus potenzielle Zielkonflikte entstehen mögen (Kadefors u. Bröchner, 2004, S. 282).

Das Assetmanagement und das hier untersuchte Propertymanagement liegen in einem Spannungsfeld: Beispielsweise möchte der Eigentümer (Investor) wenig in die Instandhaltung des Gebäudes investieren. Der Propertymanager dagegen muss neben der Eigentümer- auch die Nutzerfunktion (hierzu Pfnür, 2004, S. 295ff.) beachten und etwa eine bestimmte Infrastruktur in Bezug auf das Gebäude anbieten, damit keine Mieterfluktuation einsetzt. Darüber hinaus möchte der Propertymanager womöglich seinen eigenen leistungsabhängigen Vergütungsbestandteil erhöhen, der eventuell an eine konservative Budgetplanung gekoppelt ist. Für den Fall, dass der Eigentümer/Investor nicht mit dem Nutzer der Immobilie übereinstimmt, stellt sich die Frage, ob der Investor (Eigentümer) oder der Mieter (Nutzer) nun der Kunde des Propertymanagers ist. Bei näherer Betrachtung können beide als Kunden angesehen werden. Muir (2011), S. 43 formuliert dies wie folgt: „In general, the property manager has the ultimate responsibility for the financial and physical well-being of the property, which also includes the satisfaction and contentment of the residents, tenants, and any onsite personnel. “ Allerdings rangieren die Bedürfnisse des Investors vor denen des Nutzers, da der Investor der zahlende Auftraggeber des Propertymanagers ist (vgl. Pautmeier u.a., 2007, S.20). Oder, wie Ashley (2011), S. 105 es treffend ausdrückt: „As a real estate manager (agent) acting on behalf of the owner (principal), your words and actions are binding on the owner" [Hervorhebungen im 
Original]. Bereits diese Erkenntnis deutet auf einen möglichen Interessenkonflikt hin: Der Propertymanager muss den Auftraggeber zufrieden stellen. Zum Beispiel muss er eine stabile, hohe Mietauslastung mit zufriedenen Mietern bei zugleich kleinem Budget realisieren oder aber Mieterhöhungen durchsetzen, was wiederum die Zufriedenheit des Mieters schmälern würde. McKay (2011) weist darauf hin, dass der Propertymanager unter Umständen auch dem Mieter Informationen vorenthält und es manchmal für den Eigentümer einfacher wäre, direkt mit dem Mieter zu sprechen. Der Propertymanager verfolgt gleichzeitig aber auch eigene Interessen. Wechselt nämlich etwa eine Büroimmobilie ihren Eigentümer, so ist es wahrscheinlich, dass auch der Propertymanagement-Dienstleister ausgetauscht wird.

Der Assetmanager überträgt zur Realisierung seiner Interessen bestimmte Aufgaben und Entscheidungskompetenzen an den Propertymanager, der wiederum dafür eine Vergütung erhält (vgl. Mussnig, 2008, S.26f.). Allerdings mangelt es in der Beziehung zwischen Asset- und Propertymanager an Transparenz über die Ziele und Interessen des Vertragspartners: „An der Schnittstelle zwischen Asset- und Property-Management ist diese abweichende Einschätzung der Bedeutung von Einzelaufgaben häufig zu erkennen." (Schmitz u. Teichmann, 2010). Zwischen Assetund Propertymanager besteht eine Prinzipal-Agent-Beziehung, die sich mit Hilfe der Agenturtheorie darstellen lässt. Die Prinzipal-Agent-Beziehung ist durch zwei Charakteristika gekennzeichnet (siehe Arrow, 1985, S.37):

1. Die Handlungen des Agenten sind für den Prinzipal nicht direkt beobachtbar.

2. Das Ergebnis wird nur teilweise, aber nicht vollständig durch die Handlungen des Agenten bestimmt beziehungsweise erklärbar.

Der Agent besitzt demnach einen Informations- und Wissensvorsprung vor dem Prinzipal. Die daraus resultierenden Unsicherheiten für den Auftraggeber (Prinzipal) spielen in der Praxis beim Propertymanagement-Outsourcing eine wesentliche Rolle. Gerade vor Vertragsabschluss ist die Informationsasymmetrie von großer Bedeutung. Hierauf wird in Abschnitt 3 des Artikels näher eingegangen.

\subsection{Definition des Propertymanagements}

Wie eingangs erwähnt, existiert weder in der Literatur noch in der Praxis eine einheitliche Definition des Propertymanagements. Paradigmatisch dafür ist, dass die Begriffe nicht nur unterschiedlich verwendet werden (statt vieler Pfnür u.a., 2007 und Salustri, 2015), sondern dass sich bis heute noch nicht einmal eine einheitliche Schreibweise der Dienstleistung durchgesetzt hat. Auch darüber hinaus werden die verschiedenen immobilienwirtschaftlichen Dienstleistungen häufig nicht sauber voneinander getrennt. Beispielsweise werden in einer Studie von ERNST\&YOUNG das kaufmännische und das technische/ infrastrukturelle Objektmanagement als Kernleistungen des Assetmanagements bezeichnet (Frensch u. Fischer, 2009, S. 12). Diese Elemente sind jedoch eigentlich wesentliche Leistungen des Propertymanagements.

Die Schwierigkeiten einer klaren Begriffsbildung und trennscharfen Abgrenzung von den Schwesterdisziplinen Assetmanagement und Facilitymanagement ist nicht zuletzt darauf zurückzuführen, dass Propertymanagement in Deutschland häufig mit 
Gebäudemanagement übersetzt und daher mit dem Facilitymanagement verwechselt wird. Außerdem kann property im Englischen eine große Bandbreite von Bedeutungen haben: zunächst „Eigentum“, aber auch in der Finanzsprache „Anlagevermögen“ oder „Grund und Boden“, „bebautes Grundstück“, „Objekt“, „Immobilie“ etc. Exakt müsste es demnach Real Estate Propertymanagement heißen, doch diese Konkretisierung hat sich im Sprachgebrauch nicht durchgesetzt.

Aufgrund der genannten Herausforderungen wird das Propertymanagement in der Literatur häufig mithilfe einer Aufzählung von Tätigkeiten eines Propertymanagers konturiert. Die Definition von Fuerst etwa ist sehr allgemein gehalten und wird durch eine beispielhafte Aufzählung von Tätigkeiten konkretisiert: Propertymanagement „is the operation of commercial or residential real estate, of both leased and owneroccupied real estate. Thus, a property manager acts on behalf of the landlord in routine tasks such as rent collection, handling of leases, scheduling of maintenance and repair " (Fuerst, 2009, S. 10). Der Begriff operations, zu Deutsch Betrieb, scheint unglücklich gewählt, da es sich beim Propertymanagement - zumindest zum Teil um eine Management- und nicht um eine reine Verwaltertätigkeit handelt.

Rondeau u.a. (2006), S. 4 werden etwas konkreter und definieren wie folgt: „Property management is frequently described as the profitable operation and management of owned, leased, or subleased real property including land, buildings, assets, equipment and legal commitments for an owner, developer, or landlord. " [Hervorhebung im Original]. Für die Autoren sind der profitable Betrieb und das Management einer Immobilie Gegenstand des Propertymanagements. Auch die Leistungsempfänger, namentlich die Nutzer, Projektentwickler oder Eigentümer der Immobilie, sind in der Definition enthalten. Die Wirkungsstätte des Propertymanagements ist bei Rondeau u.a. recht weit gefasst und reicht von Grundstücken über Gebäude, Vermögensanlagen, Anlagen des Gebäudes bis zum Abschluss von bestimmten Verträgen. Diese Aufzählung scheint vor dem Hintergrund, dass das Management einer Immobilie das Management der übrigen genannten Assets einschließt, wenig zielführend.

Bogenstätter nimmt die Sicht des Qualitätsmanagements ein. Er legt für seine Definition des Propertymanagements die DIN EN 15221-1:2007-01 zu Grunde: „Property Management ist die Integration von Prozessen innerhalb einer Organisation zur Erbringung von professionellen Management-Leistungen für Immobilien, die sich nachhaltig im Bestand befinden oder befinden werden." (Bogenstätter, 2008, S.2). Auch bei Bogenstätter ist der Aspekt der Management-Leistung vorhanden. Diese wird im Gegensatz zur Definition von Rondeau u.a. als ,,professionell“ spezifiziert. Unklar ist allerdings, was mit der „Integration von Prozessen innerhalb einer Organisation“ gemeint ist. Propertymanagement ist eine Dienstleistung und muss nicht zwingend als Prozess in ein Unternehmen integriert werden, sondern kann vielmehr extern bezogen werden. Des Weiteren wird nicht recht deutlich, was mit „nachhaltig im Bestand befinden“ gemeint ist. Das Propertymanagement muss schliesslich auch für Immobilien erbracht werden, die sich nur kurzzeitig im Bestand befinden. Der Leistungsempfänger des Propertymanagements bleibt in der Definition von Bogenstätter gänzlich unerwähnt. 
Konkretisiert wird die Beschaffenheit der Propertymanagement-Leistung in der Definition von Teichmann. Er versteht unter Propertymanagement ,einen ganzheitlichen Ansatz der aktiven, ergebnisorientierten, strategischen und operativen Bewirtschaftung sowie ferner Bereitstellung und Verwertung von einzelnen Immobilien und Immobilienportfolios unter Einhaltung der Zielvorgaben des Investors beziehungsweise Eigentümers. Während üblicherweise das kaufmännische Management in Eigenleistung erbracht wird, erfolgen das technische und infrastrukturelle Management in Fremdleistung, wobei das Propertymanagement das Gebäude- beziehungsweise Objektmanagement steuert. Das im Wesentlichen kaufmännische und interdisziplinär ausgerichtete Propertymanagement übernimmt dabei die treuhänderische Wahrnehmung der Eigentümerfunktionen und ist Ansprechpartner für Nutzer und Mieter der betreuten Immobilien.“ (Teichmann, 2007, S. 19). Erstmals kommt der Aspekt des strategischen Managements ins Spiel. Allerdings spricht Teichmann von einer ,aktiven Bewirtschaftung“, anstatt den Begriff Management zu verwenden. Auch die Zielgruppe des Propertymanagements, nämlich der Investor beziehungsweise Eigentümer, wird genannt. Im Gegensatz zu den zuvor angeführten Definitionen wird der Aspekt des Fremdbezugs (von Teilen) des Propertymanagements erwähnt. Außerdem geht Teichmann darauf ein, dass der Propertymanager das Gebäude- beziehungsweise Objektmanagement steuert. Unklar ist indes, was mit der „Bereitstellung und Verwertung“ von Immobilien gemeint ist.

Kämpf-Dern baut auf der Defintion von Rondeau u. a. auf und definiert Propertymanagement als das ,,anlageorientierte, operative Management von Immobilien im Interesse des Investors während der Bewirtschaftungsphase und an den Schnittstellen zur Konzeptions-/Beschaffungs- sowie Verwertungsphase. Ziel ist die effiziente Umsetzung der vorgegebenen Objektstrategie mit Schwerpunkt auf die renditeoptimierende Bewirtschaftung der Immobilien im Investoreninteresse [Hervorhebungen im Original]." (Kämpf-Dern, 2009, S. 12 unter Berufung auf GIF, 2004a und Rondeau u.a., 2006, S. 4; siehe auch Pfnür, 2011, S.43).

In der Definition von Kämpf-Dern wird das Propertymanagement anders als in den zuvor genannten nicht auf die Haltephase beschränkt. Sie spricht wie Rondeau u. a. und Bogenstätter von Management und nicht von Bewirtschaftung. Zudem ist das Propertymanagement laut Kämpf-Dern nicht zwingend nur kaufmännisch, vielmehr umfasst das Propertymanagement auch die Nutzerperspektive, beispielsweise beim Mietermanagement. Die Definition von Kämpf-Dern enthält außerdem den Aspekt der Anlageorientierung. Die Betrachtung der Immobilien als Investitionsobjekte ist Voraussetzung für das Propertymanagement. Daher wird die Definition von Kämpf-Dern zugrunde gelegt. Auch aus der von der RICS verwendeten Definition des Propertymanagements geht hervor, dass die Investitionsziele des Eigentümers verfolgt werden sollen: „A property manager operates a real estate property for a fee often on behalf of a landlord. The property manager will commonly assist with services which include: meeting an owner's investment objectives [... “" (RICS, 2011 und RICS, 2016). 


\subsection{Leistungskatalog des Propertymanagements}

In Ermangelung einer allgemeingültigen Definition in Literatur und Praxis herrscht nicht nur hinsichtlich der Begrifflichkeiten, sondern auch in Bezug auf die Inhalte, das heißt das Leistungsspektrum des Propertymanagements betreffend, bis heute Unklarheit.

Aus dem Wesen und der Zielsetzung des Propertymanagements folgt, dass das Propertymanagement im Investoreninteresse durchgeführt wird. Die Dienstleistung wird hauptsächlich in der Haltephase der Immobilie erbracht. Daher sind ihr folgende Leistungen wesensimmanent:

- Mietermanagement (z. B. Mieterzufriedenheit)

- Management von Optimierungsmaßnahmen des Objekts (z.B. Beauftragung und Überwachung von Dienstleistern für Umbauten/Ausbauten)

- Management des Objektbudgets

Die Zielerreichung des Propertymanagements orientiert sich dabei an den Vorgaben des Assetmanagements für das einzelne Objekt. Als Bindeglied zwischen Assetund Facilitymanagement hat das Propertymanagement unter Berücksichtigung der Zielvorgaben des Assetmanagements den oder die Facilitymanagement-Dienstleister auszuwählen und zu steuern.

Im Schrifttum findet sich demgegenüber eine Reihe abweichender Leistungskataloge. Teichmann stützt seinen Leistungskatalog zum Beispiel auf die Leistungsbeschreibungen von Unternehmen, die Propertymanagement-Leistungen anbieten. Er zählt unter Berufung auf den Leistungskatalog der GIF von 2004 (GIF, 2004c) und auf mehrere Unternehmenspublikationen (Aufzählung der Unternehmen bei Teichmann, 2007, S. 20) u. a. folgende Tätigkeiten eines Propertymanagers auf (Teichmann, 2007, S. 20):

- Treuhänderische Eigentümervertretung

- Umsetzung der Immobilien- beziehungsweise Objektstrategie

- Unterstützung und Umsetzung der Maßnahmen der wertorientierten Planung, Steuerung und Kontrolle von Immobilien auf der Objekt- und Portfolioebene

- Mietmanagement

- Steuerung der operativ ausgerichteten Immobilienbewirtschaftung

- Kaufmännisches Gebäudemanagement, meist in Eigenleistung

- Technisches und infrastrukturelles Gebäudemanagement, meist in Fremdleistung

- Auswahl, Steuerung und Kontrolle des Objektmanagers

- Immobilienmarketing

- Immobiliencontrolling und Risikomanagement

- Research

Dieser Leistungskatalog zeigt zum einen eine große Bandbreite an Tätigkeiten. Zum anderen offenbart er, dass sich unter den Aufgaben eines Propertymanagers auf der einen Seite einfache Services, wie etwa die Objektbuchhaltung, und auf der anderen Seite Managementtätigkeiten, wie die Steuerung eines externen Dienstleisters für das technische Gebäudemanagement, finden. Auch Fuerst führt an, dass der Propertyma- 
Tab. 1 Aufgabenkatalog des Propertymanagements (Quelle: Kämpf-Dern, 2009, S. 12ff.)

\begin{tabular}{|c|c|}
\hline Aufgabenbereich & Aufgaben \\
\hline \multirow[t]{6}{*}{ Planung } & $\begin{array}{l}\text { Bestandsaufnahme, Analyse und Überwachung der betreuten Immobilienobjekte } \\
\text { hinsichtlich Erreichung der vom Assetmanagement vorgegebenen Objektziele } \\
\text { (z.B. Objektzustand, Vermietungs-/Leerstandsquote/Fluktuation, Mieterqualität, } \\
\text { Bewirtschaftungseffizienz, Instandhaltungsaufwand, Budgeteinhaltung) }\end{array}$ \\
\hline & $\begin{array}{l}\text { Research und Verarbeitung markt-/objektrelevanter Daten zur Erstellung von } \\
\text { Handlungsempfehlungen auf Objektebene }\end{array}$ \\
\hline & $\begin{array}{l}\text { Entwicklung und Umsetzungsplanung operativer Optimierungsmaßnahmen zur } \\
\text { Sicherstellung investorenspezifischer, öffentlich-rechtlicher und technischer } \\
\text { Vorgaben und Abstimmung mit dem Assetmanagement }\end{array}$ \\
\hline & Quantifizierung von wertsteigernden und kostensenkenden Maßnahmen \\
\hline & Operative Cash-Flow-Forecasts auf Jahres- und Mehrjahresebene \\
\hline & $\begin{array}{l}\text { Budgetplanung auf Objektebene (Vermietungsplanung, Instandhaltungs-, Mo- } \\
\text { dernisierungs-, Optimierungsplanung) }\end{array}$ \\
\hline \multirow[t]{8}{*}{$\begin{array}{l}\text { Steuerung, } \\
\text { Organisation und } \\
\text { Durchsetzung }\end{array}$} & $\begin{array}{l}\text { Auswahl, Steuerung und Controlling der Mitarbeiter sowie interner und externer } \\
\text { operativer Dienstleister (z. B. für kaufmännische, technische und infrastrukturel- } \\
\text { le Leistungen, operative Rechtsberatung, o. ä.) }\end{array}$ \\
\hline & $\begin{array}{l}\text { Laufende Analyse der Einnahmen-/Ausgabensituation sowie Identifikation, } \\
\text { Initiierung, Prüfung und Steuerung von effizienzsteigernden Maßnahmen }\end{array}$ \\
\hline & $\begin{array}{l}\text { Unterstützung von Due Diligences/Transaktionsmaßnahmen (insbesonde- } \\
\text { re Vermietung/Verkauf) durch Aufbereitung/Verfügbarmachung von objekt-/ } \\
\text { standortbezogenen Daten }\end{array}$ \\
\hline & $\begin{array}{l}\text { Vergabe, Steuerung und Controlling von Wartungs-, Instandsetzungs-, Instand- } \\
\text { haltungs- und Modernisierungsmaßnahmen }\end{array}$ \\
\hline & $\begin{array}{l}\text { Operatives Vermietungsmanagement entlang der vorgegebenen Vermietungs- } \\
\text { strategie, Umsetzung(ssteuerung) der vereinbarten Marketing- und Vermietungs- } \\
\text { maßnahmen, Betreuung von Mietern und Objektnutzern }\end{array}$ \\
\hline & $\begin{array}{l}\text { Beratung des Assetmanagements zu operativen Fragestellungen auf Objektebene } \\
\text { (Standort-, Mieterstrukturveränderungen, technische Objektveränderungen, et } \\
\text { cetera) }\end{array}$ \\
\hline & $\begin{array}{l}\text { Zusammenstellung von Einnahmen/Ausgaben beziehungsweise Kosten/Erträgen } \\
\text { von/für operative Bewirtschaftungs- beziehungsweise Optimierungsmaßnahmen } \\
\text { sowie Ermittlung der Mieterzufriedenheit }\end{array}$ \\
\hline & $\begin{array}{l}\text { Koordination und Überwachung der Miet- und Objektbuchhaltung einschließlich } \\
\text { des Zahlungsverkehrs/Mahnwesens (Miete, Nebenkosten, Kautionen, Versiche- } \\
\text { rungen, Steuern) }\end{array}$ \\
\hline \multirow{5}{*}{$\begin{array}{l}\text { Controlling, } \\
\text { Reporting, } \\
\text { Risikomanagement }\end{array}$} & $\begin{array}{l}\text { Monitoring, Analyse und Bewertung der Mieter sowie der internen und externen } \\
\text { Dienstleister im Vergleich zu vereinbarten Vertragsleistungen und -kosten }\end{array}$ \\
\hline & Monitoring und Sicherstellung der Budgeteinhaltung \\
\hline & $\begin{array}{l}\text { Analyse/Bewertung der Ergebnisse/Performance der Mitarbeiter und Dienst- } \\
\text { leister im Vergleich zur Zielvorgabe beziehungsweise einer anderen Benchmark } \\
\text { sowie Anstoßen von notwendigen Anpassungsmaßnahmen }\end{array}$ \\
\hline & Risikoüberwachung und operatives Risikomanagement auf Objektebene \\
\hline & Erstellen von Dokumentationen und Reportings an das Assetmanagement \\
\hline
\end{tabular}

nager über die Routinetätigkeiten, wie etwa die Verwaltung der Mietverträge, hinaus in Managementaufgaben involviert sei: „Property Management in the strict sense of the term is not concerned with (.) strategic tasks, however, although property mana- 
gers may be involved in these processes by collecting data and implementing policies set at the portfolio and investment management levels. " (Fuerst, 2009, S. 10).

Hier wird der Leistungskatalog gemäß Tab. 1 zugrunde gelegt, da er umfassend und in sich konsistent ist. Der Aufgabenkatalog folgt der für Management-Aufgaben maßgeblichen Einteilung in die Phasen Planung, Ausführung und Kontrolle.

\subsection{Abgrenzung zum Facilitymanagement}

Im Schrifttum werden zuweilen Propertymanagement und Facilitymanagement als eng verknüpfte Disziplinen bezeichnet: vgl. Fuerst (2009), S. 10. Cotts (2010), S. 4 et al. vertreten sogar die Auffassung, Propertymanagement sei die Bezeichnung für Faciltymanagement, sofern es sich um vermietete Objekte (in Abgrenzung zu eigengenutzten Immobilien) handele: „In leased property, the profession [Facility Management] is titled property management [...]". In der Tat liegen beide Dienstleistungen auf einer Managementebene und namentlich auf der Objektebene. Darüber hinaus kommen beide in den gleichen Lebenszyklus-Phasen und dabei hauptsächlich in der Bewirtschaftungsphase zum Tragen, wie Abb. 2 verdeutlicht (siehe Pfnür, 2011, S.364).

Eine klare Abgrenzung beider Dienstleistungen voneinander wird indes dadurch erschwert, dass der Begriff des Facilitymanagements ebenso wie der des Propertymanagements in der wissenschaftlichen Diskussion nicht eindeutig definiert ist (siehe Pfnür, 2011, S.363, Drion u.a., 2012, S.254, Braun, 2007, S. 1 und Schulte u. Pierschke, 2000, S.34).

Schon der Begriff Facilities beziehungsweise Facilities Management ist weit gefasst und muss nicht zwingend eine Immobilie zum Gegenstand haben, sondern kann sich auch auf Organisationen beziehungsweise allgemein auf das Management von Sekundärprozessen beziehen (siehe Barrett u. Baldry, 2003, auch Schwarze, 1991, S. 209, Pfnür, 2011, S. 363 und Kämpf-Dern, 2009, S. 13f.). So definieren Barrett und Baldry Facilities Management wie folgt: „An integrated approach to maintaining, improving and adapting the buildings of an organisation in order to create an environment that strongly supports the primary objectives of that organisation." (Barrett u. Baldry, 2003, S.xi).

Hier wird das Facilitymanagement als Immobilienmanagement-Dienstleistung verstanden, die auf der Objektebene (siehe Abb. 2) stattfindet (vgl. Kämpf-Dern, 2009, S. 13f.). Die German Facility Management Organisation (GEFMA) definiert in Anlehnung an die DIN 15221 Facility Management (vgl. Stadlöder, 2012, S. 27) wie folgt: „Facility Management (FM) ist eine Managementdisziplin, welche die notwendigen Unterstützungs- (Sekundär-)Prozesse des Kerngeschäfts eines Unternehmens vereint. Dabei stehen Arbeitsplatzgestaltung, Werteerhalt und Kapitalrentabilität im Fokus des Facility Managers." (http://www.gefma.de/ definition.html) Die InTERnational FACILITy MANAGEMEnT Association (IFMA) definiert Facilitymanagement als , ,[...] a profession that encompasses multiple disciplines to ensure functionality of the built environment by integrating people, place, process and technology" (http://www.ifma.org/know-base/browse/what-isfm-). Beiden Definitionen ist gemein, dass es sich beim Facilitymanagement um eine Unterstützung von Kernprozessen handelt und die immobilienbezogenen Pro- 
zesse integrativ miteinander verknüpft werden sollen (siehe Gondring u. Wagner, 2012, S. 17, und Meng, 2016, S. 212), und Williamson u.a., 2015, S. 300f.).

Bereits aus diesen Definitionen wird der entscheidende Unterschied zum Propertymanagement deutlich: Während das Propertymanagement die finanzwirtschaftliche Perspektive einnimmt (vgl. Abb. 1 und Abschn. 2.3), erfolgt das Facilitymanagement aus dem Blickwinkel der Nutzung und des Nutzers der Immobilie. Dies konstatieren auch Rondeau et al.: „The facility professional does not usually manage corporate facilities for a profit; the mission of the person and staff is to provide high-quality, cost-effective service to in-house customers in support of the corporate business plan - that is, people and process issues rather than just place issues." (Rondeau u.a., 2006, S.4). Pointiert und zutreffend spricht daher auch Kämpf-Dern (2009), S. 14 in diesem Zusammenhang vom ,nutzungsorientierten, operativen Management immobilienbezogener Prozesse im Sinne des Nutzers" [Hervorhebungen im Original].

Neben dieses zentrale Differenzierungskriterium treten weitere Unterschiede: Namentlich werden im Schrifttum bisweilen kaufmännisches, technisches und infrastrukturelles Gebäudemanagement als Funktionsbereiche des Facilitymanagement ausgewiesen (siehe Gondring u. Wagner, 2012, S.22f. und vgl. Preuß u. Schöne, 2010, S. 60f.). Die hierunter gefassten Tätigkeiten, wie zum Beispiel die reine Durchführung der technischen Instandhaltung eines Gebäudes, weisen aber zumeist keinen Management-Charakter auf und werden in vorliegender Ausarbeitung folglich als Services verstanden. (siehe hierzu Stadlöder, 2012, S. 27). Die Facility Services müssen daher strikt von der Dienstleistung Facilitymanagement unterschieden werden (siehe hierzu Kämpf-Dern u. Pfnür, 2009, S. 25). In Abb. 2 sind diese Services als unterstützende Prozesse zuunterst verzeichnet.

\subsection{Propertymanagement als Professional Service}

Die vorangegangene Betrachtung von Definition und Leistungbestandteilen des Propertymanagements zeigt, dass in der Praxis unter dem Begriff Propertymanagement zwei grundsätzlich unterschiedliche Leistungsarten subsumiert werden: Auf der einen Seite stehen einfache Dienstleistungen wie die Objektbuchhaltung, sogenannte generic services. Auf der anderen Seite finden sich Management-Leistungen, sogenannte Professional Services, wie die Budgetplanung oder die Vergabe von Facility Services (Die dargelegte Differenzierung zwischen generic services einerseits und Professional Services andererseits beim Business Process Outsourcing findet sich bei Stauss u. Jedraßcyk (2007), S.366ff.; für eine Einordnung des Assetmanagements als Professional Services siehe Kämpf-Dern, 2010).

Was hat es genau mit den Professional Services auf sich? Eine Möglichkeit, Professional Services zu definieren, ist die Abgrenzung von einfachen, sogenannten generic services. Wie die Bezeichnung Professional Services impliziert, verfügen Professionals über eine Expertise, die durch höhere Bildung und Erfahrung zustande kommt (Hill u. Neeley, 1988, S. 18). Diese grundlegende Abgrenzung bedarf weiterer Präzisierung.

Greenwood et al. definieren Professional Service Firms als Unternehmen, „whose primary assets are a highly educated (professional) workforce and whose outputs 
are intangible services with complex knowledge" Greenwood u.a. (2005), S.661. Løwendahl et al. führen folgende Merkmale eines Professional Service an (Løwendahl u. a., 2001, S.913 und Løwendahl, 1997, S. 20):

- Die Wertschöpfung der Dienstleistung ist wissensintensiv und wird von Personen mit höherer Bildung durchgeführt, häufig verbunden mit Wissensbildung im entsprechenden Bereich.

- Die Dienstleistung basiert auf einer expertengestützten Problemanalyse.

- Die Dienstleistung zeichnet sich durch einen hohen Grad an persönlicher Einschätzung durch den Dienstleister aus.

- Die Dienstleistung ist auf jeden Kunden individuell zugeschnitten.

- Die Leistungserbringung ist durch einen hohen Grad an Interaktion mit dem Kunden gekennzeichnet.

- Die Mitarbeiter der dienstleistenden Firma haben meist einen branchenspezifischen, zertifizierten Ausbildungsgang absolviert.

- Die Mitarbeiter des Dienstleisters handeln unter Berücksichtigung professioneller Normen und priorisieren insbesondere die Kundenwünsche über den eigenen Profit.

Aus der Definition und den Merkmalen eines Professional Service ergibt sich, dass derartige Dienstleistungen kaum standardisierbar sind. Sie weisen keine Sucheigenschaften, sondern Erfahrungs- und auch Vertrauenseigenschaften auf, und lassen sich daher vor dem Kauf schwer beurteilen. Bei den zuerst genannten generic services steht die Erhöhung der Effizienz und damit die Kostenminimierung standardisierter Leistungen mit nachvollziehbaren, transparenten Prüfkriterien im Vordergrund (beispielhaft Kurzrock u. a., 2009, S. 70ff.). Im deutlichen Gegensatz hierzu zielt der Fremdbezug der Management-Leistung als Professional Service darauf, die Qualität gegenüber der Eigenerstellung zu verbessern und lässt sich deshalb allenfalls partiell durch von Service-Level-Agreements erfassen. Und selbst soweit ServiceLevels zum Einsatz kommen können, bieten sie zumeist keine Gewähr für eine erfolgreiche Outsourcing-Beziehung. Der Grund hierfür liegt in den Eigenheiten der Management-Leistung: ,, While clients can have precise specifications for manufactured goods, service level agreements between clients and Service Providers are usually vague. The quality of manufactured goods are measurable and pre-specified, whereas the quality of services is subjective and user-dependent. "(Sonmez u. Moorhouse, 2010, S. 191). Bei der Management-Leistung erwartet der Auftraggeber nicht die Bewältigung standardisierter Aufgaben, sondern darüber hinaus die Verbesserung vormals unter Umständen schlecht strukturierter Prozesse, wie etwa eine Optimierung des Mietermanagements. In Ermangelung allgemeingültiger Standards und leicht anwendbarer Ergebnismessungen, beispielsweise in Form einer Überprüfung der Erreichung festgelegter Service-Level, ist der Auftraggeber bei den Management-Leistungen dagegen auf schwer fassbare Faktoren, etwa auf das Vertrauen in den Dienstleister, angewiesen. Hinzu kommt, dass generic services - entgegen ihrer Bezeichnung - typischerweise Gegenstand eines Werkvertrags im Sinne von $\S \S$ $631 \mathrm{ff}$. BGB sind: Hier schuldet der Service Provider einen konkreten Leistungserfolg, der zudem vom Auftraggeber in aller Regel abgenommen, das heißt also kontrolliert und für gut befunden werden muss. Hinzu kommt, dass eine ,gesetzlich 
vorgesehene Haftung für Mängel [...] besteht" (siehe Busche, 2012, Rn. 14). Demgegenüber handelt es sich bei Propertymanagement um Dienstleistungen ,höherer Art", welche daher dem Recht des Dienstvertrags gemäß $§ 611 \mathrm{ff.} \mathrm{BGB} \mathrm{unterfallen.}$ Bei einem Dienstleistungsvertrag schuldet der Dienstbeauftragte allerdings nur ein Tätigwerden - und somit keinen konkreten, abnahmebedürftigen Leistungserfolg wie beim Werkvertrag (Busche, 2012, Rn. 14). Zudem besteht grundsätzlich keine Gewährleistung für Schlechtleistungen des Dienstleisters. Insoweit kann also auch der rechtliche Regelungsrahmen sowohl die Bewertbarkeit als auch die Steuerbarkeit des Dienstleisters bei Erbringung des Professional Service „Propertymanagement“, zusätzlich erschweren.

\section{Implikationen für die Outsourcing-Praxis}

Der Professional-Service-Charakter des Propertymanagements hat mannigfaltige Auswirkungen auf das Outsourcing. Hier werden die Konsequenzen in den Blick genommen, die speziell zu Beginn des Outsourcing-Prozesses, in der sogenannten Partnerwahlphase, entstehen. Tab. 2 zeigt in der linken Spalte die Kriterien eines Professional Service und in der mittleren Spalte die dazu gehörigen Ausprägungen für das Propertymanagement. Die rechte Spalte gibt einen Überblick über die existierenden Unsicherheiten und Probleme des Propertymanagement-Outsourcings auf Seiten des Assetmanagers, die im Professional Service-Charakter gründen. Der Propertymanager verfügt über einen Wissensvorsprung vor dem Assetmanager. Gerade das eingangs angesprochene, nicht eindeutig definierte Leistungsspektrum eines Propertymanagement-Unternehmens macht dem Assetmanager die Auswahl des Dienstleisters schwer. Es ist auf Seiten des Assetmanagers viel Know-how erforderlich, um den geeigneten Anbieter und die gewünschten Leistungsparameter auswählen zu können.

Wie sollte nun mit diesen Unsicherheiten in der Praxis umgegangen werden? Abb. 3 gibt einen Überblick über den Soll-Prozess des Outsourcings. Zunächst wird der Outsourcing-Gegenstand aus den Zielen des Outsourcings abgeleitet: Entweder werden die generic services und die Professional Services jeweils isoliert oder aber beide Dienstleistungsarten zusammen outgesourct. Welche Leistungsbestandteile outgesourct werden, hängt unweigerlich mit den Zielen, die der Auftraggeber mit dem Outsourcing verbindet, ab. Auf Basis des Outsourcing-Gegenstandes können in einem nächsten Schritt die benötigten Teilleistungen festgelegt werden. Und schließlich kann auch anhand der Bedeutung der einzelnen Outsourcing-Leistungen ein Anforderungsprofil erstellt werden, auf Basis dessen Dienstleister ausgewählt werden können.

Das Propertymanagement-Outsourcing ist dem Business-Process-Outsourcing zuzuschlagen, da ein gesamter Geschäftsprozess ausgelagert wird (siehe beispielhaft Mani u.a., 2010). Beim Business-Process-Outsourcing besteht typischerweise ein Zielkonflikt zwischen der angestrebten Qualitätsverbesserung einerseits und der gewünschten Kosteneinsparung andererseits (vergleiche Stauss u. Jedraßcyk, 2007, S.370ff.). Diesem sogenannten Outsourcing-Paradoxon ist durch eine klare Tren- 
Tab. 2 Unsicherheiten und Probleme aufgrund des Professional Service-Charakters beim Propertymanagement-Outsourcing

\begin{tabular}{|c|c|c|}
\hline $\begin{array}{l}\text { Merkmale eines Professional } \\
\text { Service }\end{array}$ & $\begin{array}{l}\text { Ausprägung beim Propertyma- } \\
\text { nagement }\end{array}$ & $\begin{array}{l}\text { Unsicherheiten/Probleme beim } \\
\text { Outsourcing }\end{array}$ \\
\hline $\begin{array}{l}\text { Wissensintensive Dienstleistung, } \\
\text { die von Personen mit höherer } \\
\text { Bildung/ Expertise durchgeführt } \\
\text { werden }\end{array}$ & $\begin{array}{l}\text { Interdisziplinäres Wissen not- } \\
\text { wendig, zunehmend erbracht } \\
\text { von Personen mit Studien- } \\
\text { abschluss beziehungsweise } \\
\text { Studium zum CERTIFIED PRO- } \\
\text { PERTY MANAGER }\end{array}$ & $\begin{array}{l}\text { Große Informationsasymmetrie } \\
\text { zwischen Assetmanager und } \\
\text { Propertymanager }\end{array}$ \\
\hline $\begin{array}{l}\text { Expertengestützte Problemana- } \\
\text { lyse als Basis der Dienstleistung }\end{array}$ & $\begin{array}{l}\text { Propertymanagement stützt } \\
\text { sich auf die Portfolio- und Ob- } \\
\text { jektdaten, Problemanalyse z.T. } \\
\text { von Assetmanager vorgegeben }\end{array}$ & $\begin{array}{l}\text { Unsicherheit, da schlechte } \\
\text { Kontrollmöglichkeiten der Pro- } \\
\text { blemanalyse (Wissensvorsprung } \\
\text { Propertymanager) }\end{array}$ \\
\hline $\begin{array}{l}\text { Hoher Grad an persönlicher } \\
\text { Einschätzung durch den Dienst- } \\
\text { leister }\end{array}$ & $\begin{array}{l}\text { Persönliche Einschätzung } \\
\text { des Propertymanagers im } \\
\text { Rahmen der Zielvorgaben. } \\
\text { Enge Abstimmung mit dem }\end{array}$ & $\begin{array}{l}\text { Unsicherheit, da viel Erfahrung } \\
\text { und Wissen zur Einschätzung } \\
\text { des Propertymanagers von } \\
\text { Nöten }\end{array}$ \\
\hline
\end{tabular}

Kundenindividueller Zuschnitt der Dienstleistung

Hoher Grad an Interaktion mit dem Kunden bei der Leistungserstellung

Die Mitarbeiter des Dienstleisters haben meist einen brachenspezifischen, zertifizierten Ausbildungsgang absolviert

Die Mitarbeiter des Dienstleisters handeln unter Berücksichtigung professioneller Normen und priorisieren insbesondere die Kundenwünsche über den eigenen Profit

\section{Assetmanager}

Propertymanagement ist kunden-(investoren-) und objektspezifisch
Abstimmung des Property- managers mit dem Kunden (Investor) und dem Assetma- nager/den technischen Dienst- leistern et cetera des jeweiligen Objekts

Zunehmend sind Propertymanager spezifisch ausgebildet, vergleiche etwa den Abschluss des CERTIFIED PROPERTY MANAGERS

Der Propertymanager hat die treuhänderische Eigentümervertretung zur Aufgabe. Ein Branchenverband von Propertymanagern mit verbindlichen Normen existiert nicht
Unsicherheit bezüglich der benötigter Dienstleistungsdetails

Unsicherheit in Bezug auf Involvierung von und Zusammenarbeit mit dem Dienstleister

Große Wissens- und Informationsassymmetrie zwischen Propertymanager und Dienstleister

Unsicherheit bei nicht vorhandener Leistungserfüllung

\footnotetext{
${ }^{a}$ Eigene Darstellung. Zu den Kriterien eines Professional Service siehe Løwendahl 1997, S. 20. Siehe Lüttringhaus 2015
}

nung zwischen generic und Management Services zu begegnen (vergleiche Stauss u. Jedraßcyk, 2007, S. 370ff.).

Welche Handlungsempfehlungen sind daraus abzuleiten? Die in Abschn. 2.2 angesprochene Agenturtheorie bietet ein sorgfältiges Screening im Sinne einer systematischen Informationssuche als Lösung zur Überwindung der Informationsasymmetrien vor Vertragsabschlusses. Doch welche Aspekte sollte nun ein Assetmanager bei der Auswahl eines Outsourcing-Partners berücksichtigen? Eine Studie zum Propertymanagement-Outsourcing, in der Immobilieninvestoren zu ihrem Verhalten bei der Wahl eines geeigneten Outsourcing-Partners befragt wurden (Lüttringhaus, 


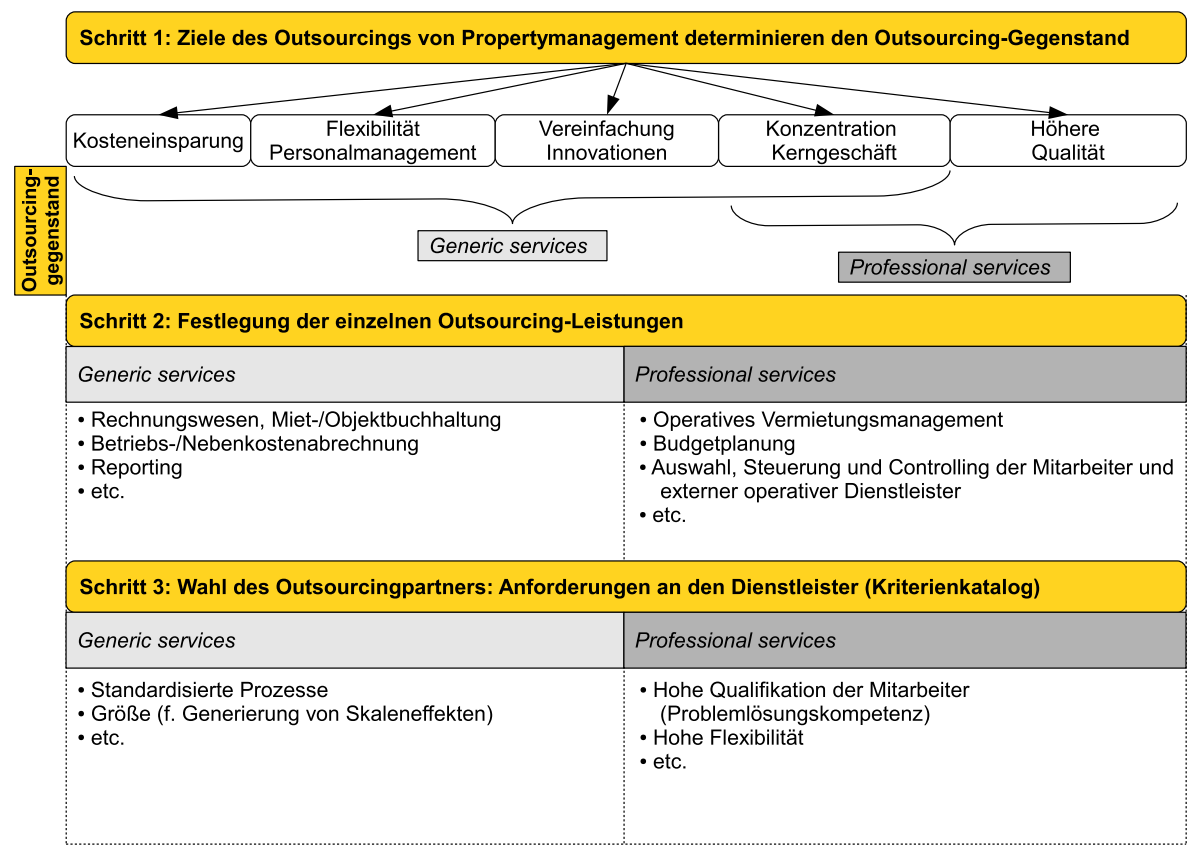

Abb. 3 Soll-Prozess der Partnerwahl des Outsourcings des Propertymanagements als Professional Service. Vereinfachte Darstellung. Eigene Abbildung. Siehe Lüttringhaus 2015

2015), hat mehrere Aspekte zur Dienstleisterwahl verprobt und eine Reihe von validen Konstrukten für die Nachfragerseite identifiziert (siehe Tab. 3). Ein bedeutender Aspekt bei der Partnerwahl ist die Reputation des Dienstleisters. Möchte sich ein Assetmanager im Vorfeld des Vertragsabschluss ein Bild von der Reputation des Propertymanagers machen, so kann er folgende Aspekte zur Entscheidung heranziehen: zum einen die eigene positive Erfahrung oder die Empfehlung eines anderen Marktteilnehmers und zum anderen eine Referenzkundenliste und ein Unternehmensranking. Ein erster Proxy für die Qualität des Dienstleistungsangebots eines Anbieters ist ein „Baukastenprinzip“, d.h. die Möglichkeit, Dienstleistungskomponenten modular zusammenzustellen. Einen zweiten Anhaltspunkt bietet das Angebot individueller Services, d.h. der Propertymanager hält eine für den Kunden maßgeschneiderte Lösung bereit. Und schließlich ist eine leistungsabhängige Vergütung ein dritter Indikator für die Qualität des Dienstleistungsangebotes. Da der Assetmanager im Rahmen eines Anbieter-Pitch schwerlich alle Mitarbeiter eines jeden Dienstleisters kennenlernen kann, benötigt er auch für die Qualität des Personals geeignete Indikatoren. Die empirische Studie verdeutlicht, dass erstens die Berufserfahrung als Propertymanager, zweitens die erfolgten Immobilienmanagement-spezifischen Weiterbildungsmaßnahmen und drittens das Auftreten der Mitarbeiter bei Erstkontakt ausschlaggebende Indikatoren sind. Proxys für das Marketing des Propertymanagers sind die Firmenpräsentation, der Internetauftritt und Marktanteil des Dienstleisters.

Die Studie liefert darüber hinaus Ergebnisse für die Anbieterseite, namentlich die Propertymanagement-Unternehmen, die im unteren Teil der Tab. 3 dargestellt 
Tab. 3 Valide Konstrukte der quantitativen Befragung von Assetmanagern zur Partnerwahl beim Propertymanagement-Outsourcing. Eigene Darstellung. Vgl. Lüttringhaus (2015)

\begin{tabular}{ll}
\hline Konstrukt & Indikatoren \\
\hline Reputation des & Eigene positive Erfahrung \\
Dienstleisters & Empfehlung eines Marktteilnehmers \\
& Referenzkundenliste \\
& Unternehmensranking \\
& „Baukastenprinzip“ \\
Dienstleistungs- & individueller Service \\
angebot & Leistungsabhängige Vergütung \\
& Berufserfahrung als Propertymanager \\
Human Resources & immobilienmanagement-spezifische Weiter- \\
& bildung \\
Auftreten der Mitarbeiter bei Erstkontakt & Firmenpräsentation \\
Marketing & Internetauftritt \\
& Marktanteil \\
Zufriedenheit mit DL allgemein \\
Kluge/Richtige Entscheidung, DL gewählt \\
zu haben \\
der Partnerwahl & Erwartungen an DL wurden erfüllt \\
Weiterempfehlungsabsicht \\
gewählten
\end{tabular}

sind. Diese sollten ihr Augenmerk auf die Zufriedenheit und Loyalität ihrer Kunden richten. Hier wird überdies deutlich, dass Qualität vor dem Preis rangiert: Zufriedene Kunden hegen eine Wiederkaufsabsicht, und es besteht ein positiver Zusammenhang zwischen Zufriedenheit und Loyalität.

\section{Fazit: Propertymanagement als Professional Service}

Entsprechend der drei Erkenntnisziele des vorliegenden Beitrags lassen sich folgende Ergebnisse festhalten:

- Erstens dient das Propertymanagement der treuhänderischen Eigentümervertretung. Die Propertymanagement-Dienstleistung wird dabei als das ,anlageorientierte, operative Management von Immobilien im Interesse des Investors während der Bewirtschaftungsphase und an den Schnittstellen zur Konzeptions-/ Beschaffungs- sowie Verwertungsphase definiert. Ihr Ziel ist die effiziente Umsetzung der vorgegebenen Objektstrategie mit Schwerpunkt auf der renditeoptimierenden Bewirtschaftung der Immobilien im Investoreninteresse [Hervorhebungen im Original]“ (Kämpf-Dern, 2009, S. 12 unter Berufung auf GIF, 2004a und Rondeau u.a., 2006, S. 4, siehe auch Pfnür, 2011, S.43). Während das Facilitymanagement stärker auf den Nutzer beziehungsweise die Nutzung des Gebäudes 
ausgerichtet ist, erfolgt das Propertymanagement primär im Interesse des Investors.

- Zweitens umfasst das Propertymanagement neben einfachen Dienstleistungen eine Reihe von komplexen Management-Aufgaben. Im Gefüge der Dienstleistungen wird das Propertymanagement daher den Professional Services zugeordnet. Propertymanagement-Unternehmen werden dementsprechend als ProfessionalService-Firms verstanden, also Unternehmen, ,whose primary assets are a highly educated (professional) workforce and whose outputs are intangible services with complex knowledge. " (Greenwood u. a., 2005, S. 661).

Aus der Definition und dem Wesen eines Professional Service folgt unmittelbar, dass asymmetrische Informationen zwischen dem Dienstleister und dem Kunden bestehen und der Kunde in hohem Maße vom Dienstleister abhängig ist (siehe beispielhaft Greenwood u. a., 2005, S. 661). Anders als bei typischen Professional Services, wie der Beratung von Unternehmen, besteht das PropertymanagementMandat nicht in der Erstellung eines Konzepts für den Auftraggeber, sondern der Propertymanager ist zu jedem Zeitpunkt in das Handeln und das Management rund um die Immobilie involviert. Er erfüllt seine Management-Leistung vor Ort. Die Einordnung des Propertymanagements als Professional Service wirkt sich auf den gesamten Vergabeprozess der Dienstleistung Propertymanagement aus: Sie schlägt sich sowohl bei der Partnerwahl als auch beim Vertragsabschluss nieder und beeinflusst schließlich die Zusammenarbeit mit dem Dienstleister ebenso wie auch dessen Steuerung.

- Drittens ergeben sich aus der vorstehenden Einordnung folgende praktische Implikationen für die Auswahl des Propertymanagers (siehe Lüttringhaus, 2015):

1. Der Assetmanager sollte das Propertymanagement als Professional Service auffassen und festlegen, ob er generic services, die Management-Leistungsbestandteile oder beides outsourcen möchte.

Die mit dem Outsourcing verbundenen Ziele und die damit in Zusammenhang stehenden Erwartungen an den Dienstleister sollten auf Basis des Outsourcing-Gegenstandes festgelegt werden. Je nachdem, ob ausschließlich generic services, ausschliesslich Management-Leistungsbestandteile oder beides outgesourct werden sollen, ergeben sich unterschiedliche Anforderungen an die Outsourcing-Partnerschaft, die von der Auswahlphase bis zum Vertragsende von Bedeutung sind. Das Outsourcing von Professional Services erfordert etwa eine höhere Qualifikation, Problemlösungskompetenz und Flexibilität auf Seiten des Dienstleisters.

2. Der Propertymanagement-Dienstleister sollte ebenfalls das Propertymanagement als Professional Service auffassen und festlegen, ob er generic services, die Management-Leistungsbestandteile oder beides anbieten möchte.

Wichtig ist, dass der Propertymanager ein klares Leistungsbild vermittelt und sich als Anbieter von generic oder Professional Services oder gegebenenfalls beiden Leistungsarten positioniert. Das Leistungsbild wirkt sich nicht zuletzt auf die Preisgestaltung aus: Die Dienstleistungsbestandteile, die den Professional Services zugeordnet werden, können grundsätzlich - da anspruchsvoller und aufwendiger - für einen höheren Preis angeboten werden als die generic services. 
3. Der Propertymanagement-Dienstleister sollte sein Augenmerk auf die empirisch bestätigten Faktoren ,Zufriedenheit mit der Dienstleisterwahl“ und „Loyalität gegenüber dem gewählten Dienstleister“ zur Verbesserung der Beziehung zu seinen Auftraggebern lenken.

Folgende empirisch bestätigte Output-Faktoren sind zu berücksichtigen:

- Zufriedenheit mit der Partnerwahl (Indikatoren: Zufriedenheit mit dem Dienstleister allgemein, richtige Entscheidung, den Dienstleister gewählt zu haben, Erwartungen an den Dienstleister wurden erfüllt)

- Loyalität gegenüber dem gewählten Dienstleister (Indikatoren: Weiterempfehlungsabsicht, Weiterbeschäftigungsabsicht, Zusatzkaufabsicht)

Eine hochwertige Dienstleistung anzubieten sollte sich demnach lohnen: Qualität rangiert bei den befragten Assetmanagern vor dem Kriterium Preis. Zufriedene Kunden hegen nämlich eine Wiederkaufabsicht, wollen also nicht zu einem billigeren Anbieter wechseln.

4. Der Assetmanager sollte auf Basis des Outsourcing-Gegenstandes und der damit verbundenen Ziele einen Kriterienkatalog für die Partnerwahl erstellen und hierfür die folgenden empirisch bestätigten Inputfaktoren heranziehen:

- Reputation des Dienstleisters (Indikatoren: eigene positive Erfahrung mit dem Dienstleister, Empfehlung des Dienstleisters durch einen Marktteilnehmer, Referenzkundenliste des Dienstleister, Unternehmensranking)

- Dienstleistungsangebot (Indikatoren: Baukastenprinzip, Individueller Service, Leistungsabhängige Vergütung)

- Human Resources (Indikatoren: Berufserfahrung als Propertymanager, Immobilienmanagement-spezifische Weiterbildung, Auftreten der Mitarbeiter bei Erstkontakt)

- Marketing (Indikatoren: Firmenpräsentation, Internetauftritt, Marktanteil)

Sowohl die immobilienwirtschaftliche Forschung als auch die Praxis können auf der hier vorgeschlagenen Qualifikation des Propertymanagements als Professional Services aufbauen.

Open Access Dieser Artikel wird unter der Creative Commons Namensnennung 4.0 International Lizenz (http://creativecommons.org/licenses/by/4.0/deed.de) veröffentlicht, welche die uneingeschränkte Nutzung, Verbreitung und Wiedergabe für beliebige Zwecke erlaubt, sofern Sie den/die ursprünglichen Autor(en) und die Quelle ordnungsgemäß nennen, einen Link zur Creative Commons Lizenz beifügen und angeben, ob Änderungen vorgenommen wurden.

\section{Literatur}

Arrow K (1985) Principals and Agents: The Structure of Business. Harvard Business School Press, Boston, S 37-51 (Kapitel 2)

Ashley B (2011) 5: THE MANAGEMENT AGREEMENT AND PLAN. In: Institute of Real Estate Management Staff (Hrsg) Principles of real estate management, 16. Aufl. Institute of Real Estate Management (IREM), Chicago, Ill., S 103-139

Barrett P, Baldry D (2003) Facilities Management. Towards Best Practice, 2. Aufl. Blackwell Science, Oxford

Bogenstätter U (2008) Property Management und Facility Management. Oldenbourg, München

Braun H-P (2007) Facility Management: Erfolg in der Immobilienbewirtschaftung, 5. Aufl. Springer, Ber$\operatorname{lin}$ 
Busche J (2012) 631 BGB. In: Säcker FJ (Hrsg) Münchener Kommentar zum Bürgerlichen Gesetzbuch, 6. Aufl. C.H. Beck, München (S. Rn. 14)

Cotts DG (2010) The Facility Management Handbook, 3. Aufl. AMACOM American Management Association, New York

Drion B, Melissen F, Wood R (2012) Facilities management: lost, or regained? Facilities 30(5/6):254-261

Frensch S, Fischer D (2009) Real Estate Asset Management. Transparenz im Leistungsdschungel. Ernst\&Young Real Estate (Studie - Fortsetzung von 2007)

Fuerst F (2009) Managing Real Estate Investments. A review of international concepts and definitions. HENLEY University of Reading (Scholarly Paper)

GEFMA Definition Facility Management/German Facility Management Association - Deutscher Verband für Facility Management e.V. http://www.gefma.de/definition.html. Zugegriffen: 30. Juni 2014

Gibson V (1994) Strategic Property Management. How can local Authorities Develop a Property Strategy? Prop Manag 12(3):9

GIF (2004) Definition und Leistungskatalog. In: Real Estate Investment Management

GIF (2004) Real Estate Investment Management/gif, Gesellschaft für Immobilienwirtschaftliche Forschung e.V., Kompetenzgruppe Real Estate Investment Management, Wiesbaden. (Studie) https:// www.gif-ev.de/onlineshop/detail/67

GIF (2004) Richtlinie. In: Real Estate Investment Management

Gondring H, Wagner T (2012) Facility Management. Handbuch für Studium und Praxis, 2. Aufl. Vahlen, München

Gondring HP, Wagner T (2016) Real Estate Asset Management. Vahlen

Greenwood R, Deephouse DL, Li SX (2005) Reputation, Diversification, and Organizational Performance of Professional Service Firms. Organ Sci 16(6):661-673

Hill CJ, Neeley SE (1988) Differences in the consumer decision process for professional vs. generic services. J Serv Mark 2(1):17-23

IFMA What is FM? Definition of Facility Management/International Facility Management Association. http://www.ifma.org/know-base/browse/what-is-fm-1. Zugegriffen: 30. Juni 2014

Kadefors A, Bröchner J (2004) Building users, owners and service providers: new relations and their effects. Facilities 22:278-283

Kloess SG (1999) Konzeption eines Aktiven Immobilienmanagements als Dienstleistungen für institutionelle Investoren. Hochschule St. Gallen (Diss.)

Kurzrock B-M, Rottke NB, Schiereck D (2009) Factors that Influence the Performance of Office Properties. J Real Estate Portfolio Manag 15(1):59-73

Kämpf-Dern A (2008) Bestimmung und Abgrenzung von Managementdisziplinen im Kontext des Immobilien- und Facilities Management Eine Entgegnung zum gleichnamigen Beitrag von Sven A. Teichmann in der ZIÖ 2/2007. Zeitschrift Für Immobilienökonomie (ziÖ) 2008(2):59-68

Kämpf-Dern A (2010) Organisation des Immobilienmanagements als Professional Service. Performanceorientierte Konfigurationen am Beispiel des Real Estate Asset Managements. TU Darmstadt (Diss.)

Kämpf-Dern A, Pfnür A (Hrsg) (2009) Grundkonzept des Immobilienmanagements. Ein Vorschlag zur Strukturierung immobilienwirtschaftlicher Managementaufgaben./Fachgebiet Immobilienwirtschaft und Baubetriebswirtschaftslehre, Fachbereich Rechts- und Wirtschaftswissenschaften, Technische Universität Darmstadt: Arbeitspapiere zur immobilienwirtschaftlichen Forschung und Praxis. (Arbeitspapier) http://www.real-estate.bwl.tu-darmstadt.de/media/bw19/dateien/arbeitspapiere/ arbeitspapier_15.pdf

Kämpf-Dern A, Pfnür A (Hrsg) (2009) Immobilienwirtschaftliche Managementebenen und -aufgaben. Definitions- und Leistungskatalog des Immobilienmanagements/Fachgebiet Immobilienwirtschaft und Baubetriebswirtschaftslehre, Fachbereich Rechts- und Wirtschaftswissenschaften, Technische Universität Darmstadt: Arbeitspapiere zur immobilienwirtschaftlichen Forschung und Praxis. (Arbeitspapier) http://www.real-estate.bwl.tu-darmstadt.de/media/bw19/dateien/arbeitspapiere/arbeitspapier_ 15.pdf

Løwendahl BR (1997) Strategic Management of Professional Service Firms. Handelshojskolens Forlag, Copenhagen

Løwendahl BR, Revang O, Fostenlokken SM (2001) Knowledge and value creation in professional service firms: A framework for analysis. Hum Relations 54:911-931

Lüttringhaus S (2015) Outsourcing des Propertymanagement als Professional Service. Eine Analyse der Partnerwahlfaktoren. TU Darmstadt (Diss.)

Mani D, Barua A, Whinston A (2010) An Empirical Analysis of the Impact of Information Capabilities Design on Business Process Outsourcing Performance. Mis Q 34:39-62

McKay G (2011) Landlords \& Tenants: The Fight Club? Leader 10(1):14-17 
Meng X (2016) Facilities Management: tracing its development trajectory. Prop Manag 33(3):212-223

Muir JL (2011) 2 THE REAL ESTATE MANAGEMENT PROFESSION: POSITIONS AND RESPONSIBILITIES. In: Real Estate Management (IREM), Institute of (Hrsg) Principles of Real Estate Management, 16. Aufl. Institute of Real Estate Management, Chicago, Ill., S 39-51

Mussnig P (2008) Institutionenökonomische und Neoinstitutionalistische Erklärungsansätze. Einflüsse auf die Beratungsbranche. Verlag Dr. Müller, Saarbrücken

Oeljeschlager J (2001) Immobilienspezifisches Dienstleistungsmarketing am Beispiel von Büroimmobilien. In: Schulte K-W (Hrsg) Handbuch Immobilien-Marketing. Müller, Köln, S 245-266

Pautmeier L, Kasel R (2007) Was meint die Norm? Systematische Interpretation der ISO 9001: 2000. Qual Zuverlässigkeit (qz) 2007(52):20-23

Pfnür A, Hartmann S, Adams R (2007) Evaluierung strategischer Optionen für Geschäftsmodelle im Immobilienbestandsmanagement/Eurogroup Consulting/Forschungscenter Betriebliche Immobilienwirtschaft, TU Darmstadt. Bad Homburg: Eurogroup Consulting. (Studie - Die Studie kann angefordert werden bei Eurogroup Consulting, Bad Homburg) http://tubiblio.ulb.tu-darmstadt.de/39374/

Pfnür A (2004) Modernes Immobilienmanagement. Facility Management, Corporate Real Estate Management und Real Estate Investment Management, 2. Aufl. Springer, Heidelberg

Pfnür A (2011) Modernes Immobilienmanagement. Immobilieninvestment, Immobiliennutzung, Immobilienentwicklung und -betrieb, 3. Aufl. Springer, Heidelberg

Preuß N, Schöne LB (2010) Real Estate und Facility Management. Springer, Berlin, Heidelberg

RICS (2011) Commercial property management in England and Wales/RICS, 2. Aufl. (RICS guidance note)

RICS (2012) RICS Public Sector Property Asset Management Guidelines/Royal Institution of Chartered Surveyors. (Richtlinie, 2. Aufl.) http://www.rics.org/uk/knowledge/professional-guidance/ information-papers/public-sector-property-asset-management-guidelines/

RICS (2016) Real estat fund, asset and property management: investment services/RICS, 1. Aufl. (guidance note)

Rondeau PE, Brown KR, Lapides DP (2006) Facility Management, 2. Aufl. Wiley, New York

Salustri J (2015) A Shifting Dynamic: Asset- vs. Property Management/Institute of Real Estate Management (IREM) (White Paper)

Salustri J (2016) Added Value: Property \& Asset Management. J Prop Manag 81(1/2):44-45

Schmitz S, Teichmann SA (2010) Mehr Luft im Kontrakt. Immobilienwirtschaft 2010(5):60-61

Schulte K-W, Pierschke B (2000) Begriff und Inhalt des Facilities Management. In: Schulte K-W, Pierschke B (Hrsg) Facilities-Management. Immobilienwissen. Rudolf Müller, Köln, S 31-40 (Kapitel 1)

Schwarze J (1991) Informationsmanagement als Voraussetzung für ein erfolgreiches Facility Management. Zeitschrift Für Planung 3:209-229

Sonmez A, Moorhouse A (2010) Purchasing professional services: which decision criteria? Manag Decis 48:189-206

Stadlöder P (2012) Einheitliches FM-Verständnis. Facil Manag 2012 (1/2):26-29. (www.facility-manager. de)

Stauss B, Jedraßcyk M (2007) Business Process Outsourcing (BPO). Wertschöpfung durch externe Dienstleister. In: Bruhn M, Stauss B (Hrsg) Wertschöpfungsprozesse bei Dienstleistungen. Gabler, Wiesbaden, S 359-381 (Forum Dienstleistungsmanagement)

Teichmann S (2007) Bestimmung und Abgrenzung von Managementdisziplinen im Kontext des Immobilien- und Facilities Management. Zeitschrift Für Immobilienökonomie (ziÖ) 2007(2):5-37

Williamson C, Fearon D, Kelly K (2015) Real Estate Concepts. A Handbook. Routledge, S 281-302 (Kapitel „Property asset management“)

Wonneberger S (2009) Die Auswahl von Propertymanagement Dienstleistern - Ergebnisbericht zur empirischen Untersuchung. Arbeitspapiere zur immobilienwirtschaftlichen Forschung und Praxis 17 (www. immobilien-forschung.de) 